Revista de Antropología Social

ISSN: 1131-558X

http://dx.doi.org/10.5209/RASO 56043

\title{
El acceso y la atención sanitaria como reivindicaciones socio-políticas. Reconstruyendo la salud global desde los márgenes
}

Néstor Nuño Martínez ${ }^{1}$

Recibido: 24 de octubre de 2015 / Aceptado: 6 de septiembre de 2016

Resumen. A interpelación de organismos nacionales e internacionales, las organizaciones no gubernamentales y de base que asisten a las waria - travestis femeninos - de la ciudad indonesia de Jogjakarta han orientado la totalidad de sus programas hacia la prevención y tratamiento del VIH. A raíz de un reciente proyecto de capacitación en materia de gestión asociativa, diversos grupos de waria se han establecido como instituciones autónomas, iniciando procesos de reivindicación y negociación socio-política enfocados a la obtención de acceso y atención sanitaria gratuitos. A través de un análisis de las diferentes motivaciones y consecuencias que subyacen a estos procesos, este artículo busca refutar y repensar las dinámicas que actualmente preponderan en el campo de la salud global, caracterizadas por construir y presentar la lucha contra el VIH y la Malaria como las imperiosas prioridades médicas en el Sur Global.

Palabras clave: Salud global; waria; derecho a la salud; Indonesia; neo-liberalismo.

\section{[en] Health access and medical care as socio-political vindications. Rethinking global health from the margins}

\begin{abstract}
According to the exigencies of national and international institutions, non-governmental and grassroots organizations assisting waria - male-to-female transvestites - in the Indonesian city of Jogjakarta have focused all their programs in the prevention and treatment of HIV. As a result of a recent training program in social management, groups of waria have decided to establish themselves as independent institutions and initiate socio-political processes of revindication and negotiation aimed at obtaining free medical access and care. Throughout analysing the underlying causes that have motivated these practices and their consequences, this paper seeks to discuss and rethink the prevailing priorities constructed in global health - characterized by presenting the battle against HIV and Malaria as a the pressing health priorities in the Global South.
\end{abstract}

Keywords: Global health; waria; right to health; Indonesia; neo-liberalism.

Sumario. 1. Introducción. 2. El acceso a la salud como problema socio-sanitario. 3. El comienzo del cambio: las reivindicaciones socio-políticas de las waria entorno a la salud. 4. Los desafíos más allá del acceso médico. 5. Discusión: neo-liberalismo y derecho a la salud. 6. Conclusión. 7. Referencias bibliográficas.

Cómo citar: Nuño Martínez, N. (2017). El acceso y la atención sanitaria como reivindicaciones socio-políticas. Reconstruyendo la salud global desde los márgenes, en Revista de Antropología Social 26(1), 73-91.

Instituto de Medicina Tropical y Salud Pública de Suiza.

nenunom@gmail.com 


\section{Introducción}

En la década de 1990, las prácticas neo-liberales propuestas por el consenso de Washington y promovidas por organismos multilaterales como el Banco Mundial o el Fondo Monetario Internacional demostraron no ser eficaces a la hora de abordar las desigualdades a nivel mundial. Esto se tradujo en un empeoramiento de la situación política, económica, social y sanitaria de aquellos países que aplicaron dichos principios de manera más pragmática (Hanlon, 1996; Homedes y Ugalde, 2005; Keshavjee, 2015; Prats, 2006; Sanahuja, 2007). La crisis de los paradigmas economicistas que hasta el momento habían guiado los programas de desarrollo dio pie a que otros organismos como las Naciones Unidas — que hasta la fecha había ejercido un rol consultivo para los países - pasaran a diseñar y coordinar políticas y programas de desarrollo a nivel mundial (Alonso, 2012; Pérez, 2012; Sanahuja, 2007: 77). La cuestión del desarrollo pasó de ser un asunto de orden "nacional" a convertirse en una "agenda global", diseñada con el fin de organizar y movilizar diferentes acciones y procesos que presentaban la salud y el enfoque de las capacidades humanas (Sen, 1989) como ejes centrales para asegurar el "progreso" y la erradicación de la "pobreza" y las desigualdades en el mundo (Naciones Unidas, 2000).

En los quince años que han transcurrido desde este cambio de paradigmas, el contexto mundial en el ámbito del desarrollo se ha transformado de manera significativa. La Ayuda Oficial al Desarrollo ha ido progresivamente perdiendo importancia en detrimento de otros flujos económicos como los créditos de exportación, las inversiones privadas y las remesas familiares; la incidencia de las enfermedades infecciosas y transmisibles se ha ido reduciendo, dando paso a un incremento de patologías crónicas y degenerativas a nivel mundial; por último, las poblaciones más vulnerables - desde una perspectiva socio-económica - han pasado a concentrarse de manera sustancial en las ciudades de los denominados "países de rentas medias", aumentando la desigualdad y los procesos y dinámicas de exclusión y marginalización (Arce y Long, 2000; Chandy y Gertz, 2011; Naghavi, Wang, Lozano et al., 2014; Sumner y Lawo, 2013). Paralelamente, este periodo de transiciones económicas, epidemiológicas y demográficas ha dado lugar a una reformulación de los fundamentos que construyen las políticas, prioridades e intervenciones en el campo de la salud internacional; este cambio ha sido denominado salud global. En palabras de Craig Janes y Kitty Corbett (2008), la salud global es un campo multidisciplinar que busca entrelazar las nociones de salud y el bienestar con procesos "globales", concebidos como dinámicas replicadas en distintos lugares del mundo y caracterizados por tener un marcado carácter descontextualizador y reinterpretativo de las realidades locales. Estos procesos a su vez son asociados por algunos autores con la progresiva expansión de las prácticas y lógicas neo-liberales (Keshavjee, 2015; Ong y Collier, 2005). Al mismo tiempo, Mark Nichter (Nichter, 2008) asocia la noción de salud global no solo con la intersección de diversos actores - políticos, sociales, económicos - en la "globalización" de los procesos de salud/enfermedad, sino con una "ideología de responsabilidad moral" (Fassin, 2011).

2 Planteo el concepto de desarrollo como una ideología que agrupa discursos, imágenes y prácticas que buscan construir e interpretan al "otro" como un sujeto pasivo, describiendo sus limitaciones, capacidades y necesidades (Escobar, 1995; Pérez, 2012). 
En cierta medida, podría indicarse que la salud global ha representado el "retorno" del neo-liberalismo al diseño y promoción de políticas y programas de salud. Desde los últimos 25 años, la aplicación continua de diferentes prácticas y dinámicas a nivel mundial han dado lugar lo que Arachu Castro y Merrill Singer denominan "sistemas de salud insalubres" (Castro y Singer, 2004). Esta realidad sanitaria se caracteriza por un reforzamiento y perpetuación de dinámicas que propician la desigualdad en salud como son el incremento de las trabas burocráticas a la atención sanitaria, el aumento de los sobrecostos a los pacientes, la redefinición de la salud en términos de costo-efectividad y la aparición de prácticas discriminativas hacia las poblaciones más marginalizadas socialmente (Grogany Gusmano, 2007; Huffman, Veen, Hennink et al., 2012; Keshavjee, 2015; Larchanché, 2012). Este progresivo menoscabo de los sistemas públicos de salud y la promoción de una construcción de la noción de salud basada en términos de costo-eficiencia, han propiciado que el sector privado haya comenzado a conceptualizar el término como un activo de inversión y rentabilidad económica. A través de complejos entramados de relaciones y acuerdos con instituciones estatales y organizaciones no gubernamentales (ONGs), diversas industrias farmacéuticas como Pfizer, sociedades como HIVOS y GLOBALFUND y fundaciones privadas como Bill \& Melinda Gates, Doris Duke y Clinton han comenzado recientemente a centralizar parte de sus actividades en reforzar o implementar diferentes programas e iniciativas en materia de salud en el Sur Global. Algunos ejemplos son la compañía minera Gold Fields Limited, que ha promovido programas de prevención y tratamiento contra el virus de inmunodeficiencia humana (VIH) en Ghana o Guinea Ecuatorial, o la también empresa minera Antamina, la cual ha distribuido cocinas mejoradas de leña en zonas rurales del Perú con la finalidad de reducir el impacto de enfermedades respiratorias agudas como la neumonía. Por medio de la apelación de fundamentos epidemiológicos y evidencias científicas ${ }^{3}$, la inversión de grandes cantidades de dinero y la utilización de discursos que enfatizan y ensalzan las necesidades y beneficios de la salud para los mercados mundiales, la progresiva injerencia del sector privado en el campo de la salud global ha ido controlando las visiones y enfoques promovidos desde las Naciones Unidas. Este cambio se evidencia en la gradual orientación de gran parte de las intervenciones hacia patologías concretas como son la lucha contra el VIH y la Malaria (Biehl y Petryna, 2013; Matthews y Ho, 2008; McNeil, 2008; Lock y Nguyen, 2010; Pfeiffer y Nichter, 2008).

Frente a estas problemáticas y vicisitudes relacionadas con la hegemonía del conocimiento experto en la salud global, desde la antropología médica se han propuesto diferentes enfoques críticos que abogan por redefinir la salud global desde una visión más experiencial y centrada en realidades socio-sanitarias sistemáticamente silenciadas e invisibilizadas. Algunos ejemplos son el funcionamiento y las dificultades de los sistemas de salud en el Sur Global a la hora de hacer frente a la creciente incidencia mundial de pandemias como el cáncer (Livingston, 2012), el acondicionamiento y provisión de medicamentos para poblaciones vulnerables (Lo, 2013; Nguyen, 2010; Reynolds, Van der Geest y Hardon, 2003), la negociación y legitimación de los principios éticos y biomédicos presentes en los ensayos clínicos

3 Por ejemplo, en 2007 la fundación Bill \& Melinda Gates creó el Instituto para la Medición y Evaluación de la Salud (IHME en inglés), un centro de investigación cuyo trabajo busca “complementar" los datos generados por las Naciones Unidas. 
(Petryna, 2009; Towghi, 2013) o la construcción del sufrimiento como una experiencia colectiva y transformadora (Kleinman, Das y Lock, 1997). Este artículo pretende contribuir a esta reinterpretación de la salud global por medio de la descripción, análisis y replanteamiento de las necesidades socio-sanitarias de las waria de la ciudad de Jogjakarta, Indonesia.

Durante siglos, diferentes ejemplos de la transgresión de las dicotomías del género masculino/femenino han existido en Indonesia (Boellstorff, 2005; Peletz, 2006, 2011). El término waria es el neologismo actual ${ }^{4}$ utilizado para describir a los travestis femeninos $^{5}$ del país. Las waria son sujetos de cuerpo masculino que no siguen los estereotipos sociales asociados a la masculinidad, ya que usan ropa femenina (Oetomo, 2002) y se describen como "almas femeninas atrapas en cuerpos masculinos" (Boellstorff, 2004). Históricamente, las waria han sido epidemiológicamente descritas como una "población en riesgo" de transmisión y propagación de infecciones de transmisión sexual (ITS), especialmente el VIH (Ford, Siregar, Ngatimin et al., 1997; Joesoef, Gultom, Irana et al., 2003; Morin, 2008; Pisani, Girault, Gultom et al., 2004; Prabawanti, Bollen, Palupy et al., 2011). Esta categorización se ha traducido en que desde la década de 1980 todos los programas de promoción y control de la salud llevados a cabo con las waria se han ido focalizado en las ITS, atendiendo a las directrices de organismos como el programa de las Naciones Unidas para combatir el VIH (UNAIDS, 2012) o la comisión nacional indonesia para el control del VIH - KomisiPenanggulanga AIDS - (BAPPENAS, 2012).

Este artículo tiene dos objetivos principales. Por un lado, contestar críticamente la conceptualización del VIH como única e imperiosa necesidad socio-sanitaria para las waria, poniendo de manifiesto la existencia de otras prioridades que si bien son consideradas relevantes por este grupo social, son sistemáticamente silenciadas por la orientación de las políticas de salud global hacia enfermedades transmisibles e infecciosas. Por otro lado, describir de qué manera los programas de control y cuidado de la salud promovidos en las últimas décadas en el Sur Global han servido para modelar las identidades de los sujetos sociales, acercándolas a principios y valores que construyen la salud como una responsabilidad individual que debe ser demandada políticamente. El artículo está dividido en cuatro partes. En la primera se presentan las vicisitudes que obstaculizan el acceso de las waria al servicio público de salud en Indonesia. Seguidamente, se describen las reivindicaciones socio-sanitarias que determinados grupos de waria han venido realizando desde el año 2007 con el fin de revertir la situación de exclusión socio-sanitaria a la que estaban expuestas. En tercer lugar, se expone la importancia de las dinámicas de negociación que ejercen estos grupos con el fin de superar las limitaciones del acceso sanitario. Para finalizar, se discuten los resultados de la investigación dentro del marco actual de la salud global y la reivindicación del "derecho a la salud".

4 Dedé Oetomo (Oetomo, 2000: 57-58) y Tom Boellstorff (Boellstorff, 2004: 162) describen que el término fue acuñado a finales de la década de 1970 por medio de la conjunción de las palabras Indonesias wanita (mujer) y pria (hombre). Durante el artículo utilizaré el término waria para referirme tanto a su forma singular como plural y, de esta manera, mantener concordancia con su uso en el idioma indonesio, donde no existe diferenciación entre ambas formas.

5 Dentro de los estudios de género, a las waria se las categoriza como transgéneros. Sin embargo, rehúso utilizar dicho término debido a las implicaciones políticas del mismo, las cuales han implicado que la noción de transgénero pase a concebirse hoy en día como una categoría ambigua y de marcados matices etnocéntricos (Valentine, 2007). Por ese motivo, he considerado necesario emplear la terminología propuesta por Tom Boellstorff (Boellstorff, 2004). 


\section{Metodología}

La información utilizada para elaborar este artículo procede de una investigación llevada a cabo entre febrero y mayo de 2014 en la ciudad de Jogjakarta, Indonesia. En ella se realizaron un total de 35 entrevistas semi-estructuradas a miembros de ONGs y organizaciones de base, waria, médicos y personal sanitario, líderes religiosos locales y ustadz (predicadores de la fe islámica). Las entrevistas fueron llevadas a cabo en inglés y bahasa (indonesio) según la conveniencia del informante, transcritas verbatim usando el software Express Scribe y analizadas de manera sistemática por medio de categorías analíticas. También se realizaron observaciones participantes en áreas populares entre trabajadoras sexuales waria, vecindarios donde grupos waria residían, actos públicos y religiosos y dos hospitales donde había waria hospitalizadas. Finalmente se moderaron dos grupos de discusión con 10 y 7 waria respectivamente. Su análisis fue similar al de las entrevistas. Los nombres reales de los participantes en la investigación han sido sustituidos por pseudónimos con la finalidad de mantener su confidencialidad e integridad. La única excepción es la de la waria IbuMaryani. Su nombre aparece reflejado en distintas monografías (Safitri, 2013; Taha, 2012), además en vida realizó entrevistas en diarios nacionales e internacionales y grabó documentales como activista LGTB y fundadora y encargada del Pesantren Waria Senin-Kamis Al-Fatah (Internado islámico para waria).

\section{El acceso a la salud como problema socio-sanitario}

IbuMaryani había muerto. Después de llegar a mi kost ${ }^{6}$ tras haber pasado aproximadamente cuatro horas acompañándola junto a sus allegados en el hospital islámico Hidayatullah recibí un mensaje de Sunny en mi teléfono móvil en el que me comunicaba que había fallecido. Conocí a IbuMaryani a mediados del mes de febrero, cuando se encontraba ingresada en el hospital islámico Hidayatullah. Durante ese primer encuentro, me explicó la complicada situación a la que tenía que hacer frente. IbuMaryani llevaba en el hospital alrededor de mes y medio y desde entonces las facturas habían ido acumulándose hasta el punto de que la semana siguiente la iban a obligar a abandonar el hospital por no poder hacer frente a la factura médica. Hasta el momento, IbuMaryani había gastado una cantidad de 16 millones de rupias indonesias (IDR) (967,57 euros ${ }^{7}$ ) en su atención médica y, según comentaba, aún seguía debiendo dinero a su casero.

Una vez IbuMaryani salió del hospital su salud era aún delicada, lo que le impedía poder trabajar. Los días pasaron y su condición se agravó. El 17 de marzo, IbuMaryani no había podido acudir a su cita semanal en el hospital por no tener dinero suficiente para pagar el transporte en becak - popular vehículo manejado a pedales. Sus allegados se mostraban preocupados por la salud y los problemas económicos de IbuMaryani pero, sobre todo, por el poco apoyo que ella estaba

\footnotetext{
6 Alojamiento común entre la gente con bajos recursos en Indonesia. Consiste de una habitación de aproximadamente cuatro metros cuadrados dentro de una casa sin cocina. El baño suele estar situado en el exterior y es compartido entre varios kost.

7 Conversión realizada de acuerdo al cambio de moneda a fecha del 24 de septiembre de 2015. El resto de conversiones monetarias mencionadas en este artículo corresponderán al cambio de moneda en dicha fecha.
} 
recibiendo de otras waria y algunas ONGs locales con las que había colaborado en el pasado. Desde hacía muchos años, IbuMaryani se había convertido en una de las waria más populares de la ciudad de Jogjakarta. Gracias a sus esfuerzos por dar apoyo y promover la integración de las waria en la sociedad, IbuMaryani se había ganado una significativa reputación entre el resto de waria y ONGs locales, convirtiéndose en un referente para el movimiento LGTB en el país. De manera regular, waria, homosexuales y pequeñas organizaciones solían acudir a ella para pedirle dinero prestado - IbuMaryani había conseguido acumular un capital económico considerado significativo por el resto de waria- $\mathrm{y}$ otros favores como protección, cobijo o trabajo. El día 21 de marzo, IbuMaryani falleció debido a una salmonelosis sin haber recibido tratamiento.

Las waria han sido un colectivo históricamente discriminado y excluido social, política, religiosa y económicamente en Indonesia debido a su identidad de género (Boellstorff, 2004; KIWA y Toomistu, 2011; Koeswinarno, 2007; Kortschak, 2010). En la actualidad, hay determinados barrios de Jogjakarta, como el de Kricak, donde las waria no pueden entrar al ser consideradas seres pecaminosos e inmorales ante los ojos de Dios por movimientos religiosos moderados como el Nahdlatul Ulama y radicales como el Front Pembela Islam (Boellstorff, 2014; Koeswinarno, s/f; Safitri, 2013). Por lo general, las waria únicamente son aceptadas y respetadas en determinados oficios: trabajadoras sexuales, artistas de ludruk - danzas tradicionales-, músicas callejeras y estilistas (Balgos, Gaillard y Sanz, 2012; Koeswinarno, 2007; Taha, 2012). No existe un censo oficial sobre la cantidad de waria residentes en Jogjakarta, y su número oscila entre 80 y 300 . De acuerdo a los datos de la ONG local PKBI, Perkumpulan Keluarga Berencana Indonesia, - Asociación Indonesia de Planificación Familiar-, en la ciudad habría alrededor de 100 waria. Estas cifras difieren significativamente con las que manejan otras organizaciones. La ONG Komunitas AIDS Indonesia - Comunidad de seropositivos de Indonesia- calcula entre 80 y 130 waria, y los líderes de la organización Ikatan Waria Jogjakarta - comunidad waria Jogjakarta - alrededor de 300. Resulta complicado contrastar la certeza de estas informaciones debido a la constante movilidad de waria procedentes de otras regiones del país hacia Jogjakarta. Gran parte de las waria de la ciudad de Jogjakarta pertenecen al estrato social más marginal, subsistiendo en un contexto de vulnerabilidad constante (Nuño, 2016). De acuerdo a PKBI, el 60 por ciento de las waria que viven en la ciudad de Jogjakarta ejercen como trabajadoras sexuales, músicas callejeras y otros trabajos temporales, informales y precarios sin ingresos regulares. Muchas waria también indican que para poder subsitir necesitan combinar trabajos diurnos (p.eg. modelos, camareras o estilistas) y ocupaciones nocturnas, como el trabajo sexual (Hardon y Ilmi, 2014).

Esta manifiesta y prevalente subsistencia en los márgenes de la sociedad está a su vez relacionada con la dificultad que tienen las waria para poder acceder a los servicios públicos de salud. Hasta hace poco más de una década, el acceso sanitario en Indonesia debía ser pagado directamente o gestionado a través de un seguro médico privado. Actualmente, el precio por consulta en un Puskesmas (centro de atención primaria) ronda las 60.000/70.000 IDR (3,78/4,41 euros respectivamente) y en un hospital unas 200.000 IDR (12,61 euros). Un seguro privado tiene un coste aproximado de 121.000 IDR al mes - 7,56 euros. Las waria consideran estas cantidades prohibitivas teniendo en cuenta su condición socio-económica; como ejemplo, aquellas que ejercen como músicas callejeras afirman ganar alrededor de 200.000 
IDR al mes - 12,53 euros. La renta media per cápita de la población de la ciudad de Jogjakarta en 2010 fue de 249.629 IDR (15,74 euros) al mes (BPS DIY, 2010).

En 2004, el gobierno de Indonesia lanzó la primera fase de un plan llamado Akesin para promover la cobertura sanitaria universal a través de un seguro de salud obligatorio. En 2008, este seguro evolucionó hacia el actual Jamkesmas, focalizado en las poblaciones de menores recursos y que actualmente da cobertura a alrededor de 76 millones de Indonesios ${ }^{8}$. Para conseguir la cobertura del Jamkesmas, es necesario poseer una tarjeta de identidad válida en la región de residencia. Si bien las waria nacidas de Jogjakarta poseen estas tarjetas, para aquellas que a lo largo de las últimas décadas se han desplazado a la ciudad huyendo de la violencia y discriminación social y familiar en sus poblaciones de origen la obtención de esta una tarjeta supone un obstáculo significativo. El proceso de tramitación de la tarjeta implica que los familiares de la persona desplazada firmen una autorización acreditando el desplazamiento. Gran parte de las familias de las waria se niegan a firmar dicho documento debido a la vergüenza que expresan al tener una waria en la familia (Boellstorff, 2004). Por este motivo a día de hoy existe un indeterminado número de waria en la ciudad de Jogjakarta sin reconocimiento legal ni posibilidad de asistencia médica a través del Jamkesmas. Las waria también afirman que en la ciudad de Jogjakarta existen mecanismos para conseguir tarjetas de identidad falsas. Su coste suele rondar los 100 millones IDR - 6.269,52 euros.

En Jogjakarta, actualmente existen dos organizaciones que promueven programas de salud para las waria. Por un lado, la ONG PKBI lleva desde la década de 1980 centrada en la prevención de ITS, focalizándose especialmente en el VIH desde que en 1993 el primer caso de infección fuera detectado entre las waria de Jogjakarta. De acuerdo a estimaciones de PKBI, alrededor del 30 por ciento de las waria de la ciudad son seropositivas. Por otro lado, la organización de base KEBAYA, Keluarga Besar Waria Yogyakarta — gran familia waria Jogjakarta_- lleva trabajando desde 2004 en la provisión de terapia antiretroviral gratuita entre las waria seropositivas. Tras años de trabajo, estas dos organizaciones han logrado establecer un complejo entramado de acuerdos con organizaciones internacionales, el gobierno local y el sistema público de salud que permite proveer de programas gratuitos de prevención, detección y tratamiento del VIH a las waria. Gracias a estos proyectos, el impacto del VIH en las waria de Jogjakarta se ha reducido. Sin embargo todavía existen dimensiones estructurales, socio-económicas e interpersonales que no son abordadas por estos programas y que inciden de manera directa en su efectividad, como la prevalencia de un fuerte estigma social en contra de las waria seropositivas, la negativa de los clientes a utilizar condones y la desconfianza hacia las organizaciones (Nuño, 2017).

Si bien hoy en día el acceso y atención sanitaria para las waria seropositivas está asegurado, independientemente de que posean tarjeta de identidad, las waria no seropositivas sin identificación siguen teniendo que pagar para acceder a los servicios de salud. Por este motivo, en vez de acudir a centros de salud, generalmente estas waria tienden a tratar sus dolencias recurriendo a sus pares. Por medio de una descripción de la sintomatología, las waria se recomiendan mutuamente medicamentos concretos — que pueden ser adquiridos en farmacias sin prescripción médica- y las dosis que consideran suficientes en base a experiencias pasadas (Hardon y Ilmi,

8 Los beneficiarios de este programa son estimados a través de la encuesta nacional SUSENAS, que calcula los gastos de los hogares. 
2014; Hardon, Ilmi y David, 2013; Nuño, 2016). Esta es una práctica ampliamente extendida en contextos de exclusión sanitaria (Nichter, 2003; Reynolds, Van der Geest y Hardon, 2003).

\section{El comienzo del cambio: las reivindicaciones socio-políticas de las waria en torno a la salud}

Desde que el régimen dictatorial de Suharto cayó en 1998, Indonesia ha pasado por significantes cambios a nivel político, económico, social y religioso como, por ejemplo, la re-politización de las organizaciones del país (cuya actividad había sido controlada y regulada por el régimen dictatorial), y la apertura del gobierno del país a la difusión de prácticas de corte neo-liberal (Beittinger-Lee, 2010; Brenner, 2011; Hadiwinata, 2003; Nyman, 2007). Esta particularidad fue aprovechada por agencias multilaterales como el Banco Mundial para promover la creación de organizaciones de base a través de instituciones estatales y ONGs con la finalidad de "estimular" la creación de la sociedad civil indonesia ${ }^{9} \mathrm{y}$, de esta manera, "fortalecer" las relaciones entre el Estado y sus ciudadanos (Banco Mundial, 2001, 2014).

Estos cambios propiciaron que en 2006 PKBI cambiara su estrategia como institución, complementando su función asistencialista con la organización política de las waria.

PKBI cambió de estrategia, PKBI dejó de asistirlas [a las waria] como una comunidad, pero PKBI comenzó a organizarlas como una comunidad [...] y también organizamos la campaña "dame tu derecho". Las waria están perdiendo muchos derechos como ciudadanos. Normalmente nosotros decimos "son waria, son ciudadanas indonesias, merecen los mismos derechos" porque están perdiendo muchos derechos. Por eso estamos intentando que los colectivos marginalizados caminen juntos y critiquen las políticas que el gobierno hace (Entrevista con Amih, miembro de PKBI, 11 de marzo de 2014).

Entre 2006 y 2010, PKBI desarrolló un proyecto denominado "programa de desarrollo comunitario". Según sus promotores, en este proyecto se capacitó a las waria en materias como derechos humanos, participación ciudadana y gestión institucional con la intención de convertir a las waria en "ciudadanas políticamente activas". A su vez, desde PKBI se indica que asesoraron a las waria para que convirtieran los pequeños grupos informales estratificados según el origen que existían en diferentes barrios de la ciudad desde la década de 1980 en grupos de base estructurados — pero dependientes administrativa y legalmente de PKBI - para aumentar su visibilidad y poder de reivindicación ante el gobierno local. (Koeswinarno, 2007). De forma paralela a este programa, desde 2007 algunos de estos grupos compuestos por waria sin tarjetas de identidad comenzaron a formalizarse como organizaciones legales independientes reconocidas por el estado indonesio con la finalidad de adquirir un

9 Aunque el concepto ha sido ampliamente utilizado desde la década de 1990 en el campo del desarrollo y la cooperación internacional, ha resultado ser inaplicable en contextos transculturales debido a su construcción etnocéntrica y mutabilidad (Comaroff y Comaroff, 1999; Ehrenberg, 2011). 
estatus administrativo similar al de otros organismos sociales como PKBI. Esta condición permite que estos grupos concursen en programas de ayuda nacionales e internaciones y reciban financiación directa de manera autónoma. Los motivos que han desencadenado la aparición de estos grupos autónomos hay que buscarlos en las tensiones y discrepancias que se han ido tejiendo a lo largo de los años entre grupos de waria, PKBI y KEBAYA en relación a dos cuestiones principales: el acceso a la salud y la gestión y reparto de las subvenciones.

Corría el año 2007, y en Jogjakarta aún existía un vacío administrativo con respecto a los protocolos para facilitar el acceso a la salud a las waria seropositivas sin tarjeta de identidad. En ese momento, la salud de una joven waria procedente de la región de Palembang (isla de Sumatra) llamada Pursa comenzó a agravarse rápidamente. Las compañeras waria con las que convivía Pursa en uno de los grupos de la ciudad decidieron llevarla a un Puskermas, donde fue diagnosticada de sida y cáncer linfático y derivada al Hospital Público Sardjito. El personal del hospital rechazó el ingreso de Pursa al no estar inscrita en el Jamkesmas u otro seguro privado y no tener suficiente dinero para hacer frente al tratamiento; las compañeras de Pursa decidieron pedir consejo a KEBAYA. Después de analizar la situación, los líderes de KEBAYA indicaron que había una posibilidad de que Pursa fuera tratada de manera gratuita, pero era necesario que hubiera estado hospitalizada anteriormente. El grupo de waria acudió entonces a PKBI para intentar negociar la hospitalización de Pursa, pero el personal de la organización rechazó la propuesta alegando que esa acción extralimitaba su política interna. Como último recurso, las waria decidieron visitar a un pastor cristiano conocido en la ciudad por sus labores sociales para averiguar si podía ser el intermediario en las negociaciones con el director del hospital privado Bethesda para que Pursa fuera atendida sin tener que pagar en ese momento; la negociación fue satisfactoria y Pursa fue ingresada durante una semana. Cuando Pursa dejó el hospital, sus amigas acudieron a KEBAYA aunque, según sus palabras, el trato recibido por la organización no fue el apropiado. Sin notificación previa, KEBAYA compró un billete de tren y envió a Pursa a Jakarta a pesar de que ella no tenía conocidos en dicha ciudad. Cuando Pursa subió al tren su situación era grave; se encontraba semi-inconsciente y con problemas para moverse, por lo que cuando el tren llegó a Jakarta, Pursa no fue capaz de bajarse del mismo, y el tren retornó a Jogjakarta con ella en su interior. En Jogjakarta, Pursa fue expulsada del tren en la estación de Lempuyangan al no tener billete válido. Pursa estaba aún con vida, pero no era capaz de moverse y prácticamente no podía hablar. Pocas horas después murió en la estación sin haber recibido ninguna atención médica. Las amigas de Pursa culparon de lo ocurrido a KEBAYA, aunque la organización no quiso admitir las acusaciones alegando que Pursa no era miembro de KEBAYA, por lo que no tenían ninguna responsabilidad legal sobre ella. Algunas de las amigas de Pursa propusieron formular una queja formal a $L O S^{10}$ sobre la actuación de KEBAYA, pero finalmente el grueso del grupo decidió no interponer una demanda. Sin embargo, en palabras de estas waria, este acontecimiento representó un punto de inflexión para ellas. Poco tiempo después, este grupo fue el primero en comenzar los procedimientos para establecerse de manera autónoma y desligarse de las políticas y programas de PKBI y KEBAYA.

10 Una organización estatal en la que los ciudadanos pueden denunciar las malas prácticas que realizan instituciones legales. 
El hecho de que PKBI y KEBAYA hayan sido hasta hace algunos años las únicas organizaciones con potestad para recibir fondos por asistir a las waria también propició que se cuestionara la forma como gestionaban el dinero recibido:

Yo creo que en PKBI tienen un doble rasero. Por un lado ayudan a que las comunidades waria se desarrollen pero no están de acuerdo de que esto suceda sin su control. Antes de que PKBI coordinara a los grupos waria estos ya existían. Ellos pidieron financiación usando nuestros nombres, vendiendo al grupo. Eso está mal [...] También trabajé de voluntaria en PKBI y el sueldo era bastante bajo. El personal tiene buenos ingresos, 1.5 millones IDR [102,34 euros], pero para un voluntario es alrededor de 350.000 IDR [ 23,9 euros] al mes [...] Intenté hablar con ellos claramente sobre esto pero nunca escucharon. El salario es muy bajo, todo es muy caro y ya sabes, los voluntarios siempre están en la primera línea. El personal únicamente hace los reportes (Entrevista con Tika, 22 de abril de 2014).

También hay casos en los que waria se sienten discriminadas y excluidas por las propias organizaciones, ya que consideran que éstas no reparten los fondos que obtienen de manera imparcial. En 2010, KEBAYA gestionó un programa de inclusión social del estado indonesio que buscaba capacitar a waria como emprendedoras. Para ello concedió a KEBAYA la competencia para formar y distribuir 225 millones IDR (15.305 euros) entre 15 waria de la ciudad de Jogjakarta. Si bien desde el ministerio promotor el único requisito era entregar el dinero a waria con tarjeta de identidad, KEBAYA designó que únicamente daría el dinero a waria seropositivas. En palabras de representantes de la organización, esta decisión fue tomada debido a que consideraron que "las waria seropositivas necesitaban más el dinero". Sin embargo, este arbitraje desencadenó fuertes críticas entre algunas waria, propiciando que creciera el sentimiento de que las organizaciones las utilizaban únicamente buscando su propio beneficio y para conseguir más fondos (Nuño, 2017).

En la actualidad, al menos tres grupos de waria en la ciudad de Jogjakarta pueden considerarse autónomos en tanto que son instituciones legales que pueden recibir fondos, negocian directamente con el gobierno y las autoridades sanitarias sus propias propuestas y además han roto relaciones con PKBI y KEBAYA:

Nosotros comenzamos a reivindicarnos, aprendimos sobre las leyes y les recordamos lo que dice la constitución. Waria, niños de la calle y personas sin hogar deben estar incluidas en las políticas del gobierno. Entonces, comenzamos a reclamar y finalmente el gobierno aceptó nuestras demandas. Por primera vez nos dieron el Jamkesmas para 65 personas, 24 de ellas waria. Era el año 2008 (Entrevista con Vilma, 10 de marzo de 2014).

Estos grupos waria gestionan las tarjetas de identidad gratuitamente para sus miembros y también tienen la capacidad legal para expedir documentos oficiales que certifican la asistencia sanitaria sin que sea necesaria la tarjeta de identidad. El proceso es similar al que realiza KEBAYA con enfermos seropositivos: las waria que reciben esta carta firmada por el grupo deben presentarse en las oficinas del Jamkesmas, donde sus datos son contrastados con el grupo waria que expende la 
carta. Si el grupo certifica la procedencia de la carta, esa waria puede acceder a los servicios sanitarios desde ese preciso momento, recibiendo en un plazo aproximado de dos semanas su tarjeta de beneficiario del Jamkesmas. Además de la provisión de acceso médico, estos grupos también cuentan con programas gratuitos de prevención y tratamiento del VIH similares a los llevados a cabo por PKBI y KEBAYA.

\section{Los desafíos más allá del acceso sanitario}

El éxito de estos grupos autónomos a la hora de conseguir un acceso médico gratuito para sus miembros está motivando que waria de otras ciudades y regiones como Palembang, Medan o Bali se trasladen a vivir a Jogjakarta y se unan a dichos grupos con la finalidad de conseguir tarjetas de identidad y acceso médico gratuito:

El último lugar donde estaba trabajando era Jakarta. Tenía el trabajo asegurado en un salón de belleza [...] entonces Vilma me llamó y me dijo que tenía que volver a Jogjakarta a hacerme una tarjeta de identidad. Para hacerme la tarjeta necesitaba como siete días más o menos, pero mi jefe solo me daba dos días libres. Entonces ahí tenía dos opciones, o volver a Jogjakarta y conseguir mi tarjeta de identidad o quedarme en Jakarta y no conseguir una por el resto de mi vida. Decidí dejar el trabajo e ir a hacerme mi tarjeta de identidad (Entrevista con Sandi, 4 de abril de 2014).

El Jamkesmas ofrece un paquete de coberturas que únicamente incluye atención hospitalaria, ambulatoria, materna y preventiva. Además, sus beneficiarios solamente tienen derecho a la cobertura de medicamentos básicos prescritos por los facultativos. Otro tipo de gastos extraordinarios y tratamientos de considerable costo económico deben ser pagados por los pacientes. A través de los años, los grupos autónomos de waria han desarrollado dinámicas de negociación y presión colectiva ante las autoridades y el personal sanitario con la finalidad de superar estas limitaciones del Jamkesmas y hallar la mejor forma de seguir asegurando tratamientos totalmente gratuitos para sus miembros.

A finales de 2012, una waria llamada Shasa que vivía en la región Indonesia de Batam comenzó a perder su visión a causa de una toxoplasmosis ${ }^{11}$, y el facultativo la recomendó desplazarse a Jakarta debido a que el acceso al tratamiento resultaba complicado en Batam. Shasa se desplazó a Jakarta, y al acudir al hospital descubrió que el Jamkesmas no cubría su tratamiento. El precio de los medicamentos para tres meses rondaba los 19 millones IDR (1.229,31 euros), un dinero que Shasa no podía pagar ya que subsistía gracias al apoyo y la ayuda que le brindaban grupos de waria que la cuidaban. Al no poder comenzar su tratamiento, Shasa se quedó totalmente ciega. Era el verano de 2013, y una amiga waria que había sido tratada en Jogjakarta gracias a la intermediación de uno de los grupos autónomos de la ciudad recomendó a Shasa desplazarse. Shasa se unió al grupo waria que le había recomendado su amiga y las waria de este grupo le hablaron a Shasa sobre la existencia de un subprograma estatal que procuraba de forma gratuita aquellos tratamientos no cubiertos por

11 La Toxoplasmosis es una infección causada por el parásito Toxoplasma Gondii. El contagio puede producirse a través del contacto directo con animales (p.eg. gatos) o la ingesta de carne cruda o poco cocinada. 
el Jamkesmas. Para ello únicamente era necesario requerirle al facultativo médico la redacción de una carta para el director financiero del hospital explicando el caso concreto del paciente. Al acudir al Hospital Sardjito, el doctor que examinó a Shasa indicó que no podía iniciar el tratamiento ya que había pasado demasiado tiempo desde su ceguera, sugiriendo a Shasha una operación. Pasaron las semanas y el doctor aún no había aprobado la intervención, por lo que las waria propusieron organizar una reunión con el director del Hospital Sardjito para poner en su conocimiento el caso de Shasa. Esta es una práctica que las waria de este grupo describen como frecuente y necesaria para agilizar los trámites administrativos.

Esta potestad de negociación que estos grupos autónomos han ido adquiriendo con el paso de los años no solo ha implicado que waria de todo el país describan la ciudad de Jogjakarta como un "océano seguro" — lautamam — para tratar sus dolencias, sino que ha ayudado a crear y establecer redes y relaciones con otros grupos waria del país:

Tiara: Honestamente, Gila es también una activista. Ella puede llevar a cabo un programa que nosotros no podemos, por lo tanto yo he aprendido de ella cómo desarrollarlo. En compensación le he enseñado qué es lo que hacemos aquí. También la necesitamos para esto.

Gila: [...] yo sé que de donde yo vengo [Batam] lo puedo hacer, pero aquí creo que aún no se puede. Actualmente existe un programa estatal en el que si un paciente necesita moverse a otro lugar para ser tratado, el gobierno paga su billete y el de su acompañante y también el coste de vida en el nuevo sitio (Entrevista con varias waria, 3 de abril de 2014).

Por medio del intercambio de experiencias diferentes, grupos de waria del país están comenzando a desarrollar estrategias y propuestas de coordinación con la finalidad de confrontar la vulnerabilidad y discriminación sanitaria que aún sufren en todo el país.

\section{Discusión. Neo-liberalismo y derecho a la salud}

Los resultados expuestos en este artículo describen de qué manera las waria de la ciudad de Jogjakarta han buscado mecanismos y estrategias para superar la discriminación socio-sanitaria que sufrían, principalmente por la dificultad para conseguir tarjetas sanitarias. Estas dinámicas y procesos ponen de manifiesto diferentes cuestiones y paradojas. Si bien el Jamkesmas pretendía ser una excepción a las prácticas y dinámicas neo-liberales que imperan actualmente en Indonesia (Hadiz y Robinson, 2006) al haber sido creado con el propósito de aumentar la cobertura sanitaria a las poblaciones más vulnerables del país, el análisis de la situación socio-legal de las waria refleja lagunas burocráticas que, paradójicamente, han terminado excluyéndolas del programa a pesar de ser una de las poblaciones que deberían beneficiarse del mismo. Paralelamente, el diseño del Jamkesmas evidencia una de las principales discusiones que este tipo de programas de salud - centrados únicamente en el acceso a los servicios sanitarios - ha suscitado en los últimos años. Si bien el acceso 
es universal, la cobertura no contempla el hecho de que las poblaciones más vulnerables tienden a ser más propensas a desarrollar coinfecciones y enfermedades de complicado tratamiento debido, justamente, a su propia condición (Singer, Erickson, Badiane et al., 2006). Esta es una dinámica generalmente replicada en los modelos de salud modelados por el neo-liberalismo (Castro y Singer, 2004). Por otro lado, las narrativas descritas ejemplarizan también uno de los contrasentidos que, tristemente, se han venido generalizando dentro del campo de la salud global en los últimos años. ONGs y organizaciones de base que, a priori, deberían focalizar su atención en aquellas realidades socio-sanitarias con una incidencia significativa a nivel local al poseer un mayor conocimiento y contacto con dicha realidad (Appadurai, 2000), han tendido progresivamente a centrarse en aquellas "prioridades" construidas de manera vertical desde instituciones internacionales, pasando a deslegitimar y silenciar otras realidades de aquellos grupos a los que supuestamente ayudan. En conversaciones con diversos miembros de PKBI y KEBAYA, todos ellos declararon ser conscientes del problema que existe entre las waria no seropositivas para conseguir acceso a la salud. Sin embargo, también argumentaban que este interés y esfuerzo por desarrollar estrategias de prevención y tratamiento para el VIH les resultaba "lógico" ya que consideraban más "sencillo" conseguir financiación de organismos internacionales (dentro de un contexto que describían como muy competitivo) para promover programas de VIH que centrarse en otras áreas como los micro-créditos, descritos como menos "atractivos" para los donantes. Otro claro ejemplo que pone de manifiesto esta discrepancia de prioridades entre instituciones y waria en Jogjakarta es la dificultad y precariedad para el acceso y consumo de tecnologías de modificación corporal como silicona u hormonas femeninas y la negativa de PKBI a proveer estos servicios a pesar de disponer de medios y recursos para ello (Nuño, 2016).

Estas decisiones institucionales, construidas a través de un prisma racionalista, tienden a ser interpretadas a ojos de las poblaciones asistidas como reproductoras de las desigualdades, intereses economicistas, jerarquías sociales y políticas discriminatorias que prevalecen en sus sociedades (Aldaba, Antezana, Valderrama et al., 2000; Ashraf, 2013).

Los hechos detallados en este artículo no solo ponen de manifiesto esta discrepancia entre prioridades propuestas desde la salud global y las necesidades localmente construidas, sino que también evidencian el impacto de la "globalización de la salud". Si bien antaño los programas de promoción y control de la salud en el Sur Global eran contingentes, la inferencia de las Naciones Unidas a la hora de transformar la salud en una cuestión "global" ha generalizado la diseminación de las lógicas y dialécticas de la salud, reconfigurando la forma en la que los sujetos sociales perciben e interpretan dicha noción. Este hecho se observa claramente en el patrón que han mantenido las demandas de las poblaciones que subsisten en la marginalidad en el Sur Global en los últimos años, las cuales han ido progresivamente desligándose de movilizaciones de corte económico o social para centrase principalmente en la reivindicación de la salud como derecho (Fassin, 2010). La progresiva expansión del neo-liberalismo en el campo de la salud global no solo ha tenido un impacto en el ámbito institucional, sino que también ha servido para modelar nuevas identidades fundamentadas en valores que describen la libertad, el individualismo y la iniciativa personal como elementos centrales para la emancipación y el bienestar de los sujetos (Rose, 1999). Desde la década de 1970, diferentes ONGs y organizaciones humanitarias comenzaron a diseñar programas y actividades de capacitación 
en el Sur Global que fueran "vehículos" para la promoción de estas identidades y valores que abogaran por construir la salud como una responsabilidad individual que debe ser gobernada y regulada (Keshavjee, 2015; Kleinman, Das y Lock, 1997; Lock y Nguyen, 2010). Consiguientemente, diferentes grupos sociales en la marginalidad, influenciados por estas dinámicas han pasado en los últimos años a promover movilizaciones socio-sanitarias con la finalidad de protestar en contra de diferentes escenarios que consideraban discriminatorios en términos de salud, evocando para ello principios como el de la "ciudadanía biológica" que explora la autora Adriana Petryna (2002). Estas movilizaciones tienen como principal objetivo la exigencia del derecho a la salud o la reivindicación identitaria en base a una condición bio-sanitaria. Alrededor del mundo se pueden observar ejemplos de este tipo de movilizaciones entre pacientes seropositivos en Brasil y Sudáfrica (Biehl, 2004; Fassin, 2007), personas con distrofia muscular en Francia (Rabeharisoa, 2006), supervivientes de la tragedia de Chernóbil (Petryna, 2002) o en el acceso a operaciones estéticas en Brasil (Edmonds, 2007).

Según Jane Cowan (Cowan, Dembour y Wilson, 2001) este discurso de la "cultura de los derechos" tiene su origen en el énfasis de organismos como las Naciones Unidas en la promoción de los derechos humanos y los acuerdos y declaraciones de corte universalista. Este uso de los derechos como discursos estructurados y legitimadores resulta 'convincente' y 'apropiado' en tanto que en muchas sociedades el racionalismo basado en la construcción de evidencias y convencionalismos legales se ha convertido en uno de los motores principales de las reivindicaciones. A su vez, estos discursos encarnan dimensiones y dinámicas que proponen construcciones de la subjetividad y de las relaciones sociales en un contexto universalizado. Si bien estos mecanismos resultan útiles para reivindicar problemáticas históricamente invisibilizadas como las que se han analizado en este artículo, también tienen sus limitaciones. Por ejemplo, la reivindicación del 'derecho a la salud' promueve una construcción individualista de los derechos y aborda el sufrimiento a través de estrategias legales y técnicas en vez de basarse en marcos éticos y morales. A su vez, únicamente se centran en el análisis de aspectos concretos de la experiencia humana sin proponer una visión holística y no etnocéntrica del bienestar y las necesidades (Butt, 2000).

\section{Conclusión}

La progresiva intrusión del sector privado en la salud global ha dado lugar a que patologías como la Malaria o el VIH hayan pasado a ser construidas como 'prioridades imperantes' a nivel mundial, silenciando otras realidades socio-sanitarias con un fuerte impacto a nivel local. En el caso de las waria - travestis femeninos - de Indonesia, el exclusivo énfasis que han puesto las organizaciones no gubernamentales y de base que asisten a este colectivo en la ciudad de Jogjakarta en programas de prevención y tratamiento del VIH ha generado importantes malestares y contrariedades ya que otros problemas de índole socio-sanitarios, como la exclusión en los servicios públicos de salud, no son abordados. A raíz de un programa de desarrollo comunitario, los grupos de waria que percibían de forma más marcada esta discriminación decidieron romper relaciones con los colectivos que hasta el momento las habían asistido, constituyéndose como organizaciones autónomas con el fin de 
escudriñar estrategias de reivindicación y negociación del acceso y atención sanitarios. El desarrollo de estas movilizaciones ha puesto de manifiesto la fragilidad del Jamkesmas, el programa de atención sanitario a las poblaciones más vulnerables, y ha evidenciado la importancia que tienen estos grupos de waria a la hora de negociar con las instituciones y los facultativos médicos una atención sanitaria gratuita y sin restricciones. La asociación de estos procesos dentro del marco neo-liberal que actualmente prepondera en las políticas y programas de salud en el Sur Global evidencia dos claros desenlaces. Por un lado, la discrepancia entre las orientaciones en salud diseñadas y promovidas desde un enfoque vertical y las necesidades sociosanitarias que se manifiestan a nivel local. Por otro lado, la dirección de las movilizaciones de los grupos que subsisten en los márgenes de la sociedad hacia estrategias que buscan reivindicar la salud como derecho.

\section{Referencias bibliográficas}

Aldaba, Fernando; Antezana, Paula; Valderrama, Mariano; et al. (2000). "NGO strategies beyond aid: perspectives from Central and South America and the Philippines". Third World Quarterly, vol. 21, no. 4: 669-683.

Alonso, José Antonio (2012).“La teoría del desarrollo y los cambios en el sistema internacional”. Revista CIDOB, 100: 43-65.

Appadurai, Arjun (2000). "Grassroots Globalization and the Research Imagination". Public Culture, 12(1): 1-19.

Arce, Alberto; Long, Norman (2000). Anthropology, Development and Modernities. Exploring discourses, counter-tendencies and violence. Londres y Nueva York: Routledge.

Ashraf, HM A (2013). “'Why you can't pay if you can eat?': tales of how Women Encounter Unpleasant NGO Practices in Bangladesh”. Student Anthropologist, vol. 3, issue 2: 7-26.

Banco Mundial

- (2001). Indonesia - Country Assistance Strategy. Washington, DC: World Bank. Último acceso, 2 de junio de 2014: http://documents.worldbank.org/curated/en/2001/02/828313/ indonesia-country-assistance-strategy

- (2014). Evolution and Challenges of Civil Society Organizations in Promoting Democratization in Indonesia. Paper presented at the Supporting Constructive Engagement between Government of Yemen and CSOs Conference. Último acceso, 2 de junio de 2014: http://www.worldbank.org/content/dam/Worldbank/Event/MNA/yemen_cso/english/ Yemen_CSO_conf_Pres_Indonesia_ENG.pdf

BAPPENAS (2012). Laporan pencapalian tujuan pembangunan millennium di Indonesia 2011. Gobierno de Indonesia.

Balgos, Benigno; Gaillard, J.C.; Sanz, Kristinne (2012). "The warias of Indonesia in disaster risk: The case of the 2010 Mt Merapi eruption in Indonesia". Gender \& Development, vol. 2(2): 337-348.

Beittinger-Lee, Verena (2010). (UN) Civil Society and Political Change in Indonesia: A Contested Arena. Nueva York: Routledge.

Biehl, João (2004). "The Activist State: Global Pharmaceuticals, AIDS, and Citizenship in Brazil". Social Text 80, 22(3): 105-32.

Biehl, João; Petryna, Adriana (Eds.) (2013). When People Come First: Critical Studies in Global Health. Princeton University Press. 
Boellstorff, Tom

- (2004). "Playing Back the Nation: Waria, Indonesian Transvestites". Cultural Anthropology, vol. 19, issue 2: 159-195.

- (2005). The gay archipelago: Sexuality and Nation in Indonesia. Princeton: Princeton University Press.

- (2014). "Lessons from the Notion of 'Moral Terrorism"”, en Thomas Stodulka y Birgitt Röttger-Rössler (Eds.), Feelings at the Margins: Dealing with Violence, Stigma and Isolation in Indonesia. Chicago: University of Chicago Press, 148-158.

BPS DIY (2010). Daerah Istimewa Yogyakarta Dalam Angka. Subdivision Regional de Análisis Estadístico, Provincia de Yogyakarta.

Brenner, Suzanne (2011). "Private Moralities in the Public Sphere: Democratization, Islam, and Gender in Indonesia". American Anthropologist, vol. 113(3): 478-490.

Butt, L. (2002). "The Suffering Stranger: Medical Anthropology and International Morality”. Medical Anthropology Quarterly, 21: 1-24.

Castro, A.; Singer, M. (Eds.) (2004). Unhealthy Health Policy - a Critical Anthropological Examination. Maryland: Altamira Press Chandy, L.; Gertz, G. (2011). Two Trends in Global Poverty. The brookings institution.

Comaroff John L.; Comaroff, Jean (Eds.) (1999). Civil Society and the Political Imagination in Africa. Chicago: University of Chicago Press.

Cowan, Jane K.; Dembour, Marie-Bénédicte; Wilson, Richard, A. (Eds). (2001). Culture and Rights. Anthropological Perspectives. Cambridge University Press.

Edmonds, Alexander (2007). "The Poor have the Right to Be Beautiful': Cosmetic Surgery in Neoliberal Brazil”. The Journal of the Royal Anthropological Institute, 13 (2): $368-81$.

Ehrenberg, John (2011). "The History of Civil Society Ideas", en Michael Edwards (Ed.),The Oxford Handbook of Civil Society. Nueva York: Oxford University Press, 15-25.

Escobar, Arturo (1995). Encountering Development. New Jersey: Princeton University Press.

Fassin, Didier

- (2007). When Bodies Remember: Experiences and Politics of AIDS in South Africa. Berkeley: University of California Press.

- (2010). "El irresistible ascenso del derecho a la vida. Razón humanitaria y justicia social". Revista de Antropología Social, vol. 19: 191-204.

- (2011). Humanitarian Reason: A Moral History of the present. Berkeley: University of California Press.

Ford, Nicholas; Siregar, Kemal; Ngatimin, Rusli; et al. (1997). "The hidden dimension: sexuality and responding of threat of HIV/AIDS in South Sulawesi, Indonesia". Health \& Place, vol. 3(4): 249-258.

Grogan, Colleen M.; Gusmano, Michael K. (2007). Healthy Voices, Unhealthy Silence. Advocacy and Health Policy for the Poor. Washington D.C.: Georgetown University Press.

Hadiwinata, Bob S. (2003). Politics of NGO in Indonesia: Developing Democracy and Managing a Movement. Londres y Nueva York: Routledge.

Hadiz, Vedi R.; Robinson, Richard (2006). "Neo-liberal Reforms and Illiberal Consolidations: The Indonesian Paradox" en Kevin Hewison y Richard Robinson (Eds.), East Asia and the Trials of Neo-Liberalism. Londres:Routledge, 24-46.

Hanlon, Joseph (1996). Mozambique: Who Calls the Shots? Londres: James Currey.

Hardon, Anita; Ilmi, Nurul I. (2014). "On Coba and Cocok: youth-led drug-experimentation in Eastern Indonesia”. Anthropology \& Medicine, vol. 21, no. 2: 217-229. 
Hardon, Anita; Ilmi, Nurul I; David, Takeo H. (2013). “Chemical Sexualities: The Use of Pharmaceutical and Cosmetic Products by Youth in South Sulawesi, Indonesia". Reproductive Health Matters, 21(41): 214-24.

Homedes, N.; Ugalde, A. (2005). "Why neoliberal health reforms have failed in Latin America". Health Policy,71(1): 83-96.

Huffman, Samantha A.; Veen, Jaap; Hennink, Monique M.; et al. (2012). "Exploitation, vulnerability to tuberculosis and access to treatment among Uzbeklabor migrants in Kazakhstan". Social Science \& Medicine, 74:864-872.

Janes, Craig R; Corbett, Kitty K. (2009). “Anthropology and Global Health”. Annual Review of Anthropology, 38: 167-83.

Joesoef, M. R.; Gulrom, M.; Irana, I.D.; et al. (2003). "High rates of sexually transmitted diseases among male transvestites in Jakarta, Indonesia". International journal of STD \& AIDS, vol. 14(9): 609-13.

Keshavjee, Salmaan (2015). Blind Spot: How Neoliberalism Infiltrated Global Health. University of California Press.

Kiwa; Toomitsu, Terje (2011). Wariazone. Documental independiente sobre las waria de Indonesia.

Kleinman, Arthur; Das, Veena; Lock, Margaret (2007). Social Suffering. Berkeley: University of California Press.

Koeswinarno, Oleh

- (2007). Kehidupan Bergama Waria Muslim di Yogyakarta. Yogyakarta: Universitas Gadjah Mada.

- (S/F). "Islam and Marginalization of Waria. An effort to reconstruct Islamic Law about Transgender". Artículo sin publicar.

Kortschak, I. (2010). Invisible People: Poverty and Empowerment in Indonesia. Jakarta: The Lontar Foundation.

Larchanché, Stéphanie (2012). "Intangible obstacles: Health implications of stigmatization, structural violence, and fear among undocumented immigrants in France". Social Science \& Medicine, 74: 858-863.

Livingston, Julie (2012). Improvising Medicine: An African Oncology Ward in an Emerging Cancer Epidemic. Durham: Duke University Press.

Lo, Marieme S. (2013). “Confidant par excellence, advisors and healers: women traders' intersecting identities and roles in Senegal". Culture, Health \& Sexuality, vol. 15, suppl. 4: S467-S481.

Lock, Margaret; Nguyen, Vinh-Kim (2010). Anthropology of Biomedicine.Oxford: WilleyBlackwell.

Naciones Unidas (2000). United Nations Millennium Declaration. Nueva York, sesión 55 del 18 de septiembre. Último acceso 23 de septiembre de 2015: http://www.un.org/ millennium/declaration/ares552e.pdf

Naghavi Mohsen; Wang,Haidong; Lozano, Rafael; et al. (2014). “Global, regional, and national age-sex specific all-cause and cause-specific mortality for 240 causes of death, 1990-2013: a systematic analysis for the Global Burden of Disease Study 2013”. Lancet, 385: 117-71.

Nguyen, Vihn-Kim (2010). The Republic of Theraphy. Duke University Press.

Nichter, Mark

- (2003). "Harm Reduction: A Core Concern for Medical Anthropology", en Barbara Herr Harthorn y Laury Oaks (Eds.), Risk, Culture and Health inequality: Shifting Perceptions of Danger and Blame. Connecticut: Praeger Publishers Wesport, 13-36. 
- (2008). Global Health: Why Cultural Perceptions, Social Representations, and Biopolitics Matter. The University of Arizona Press.

Nuño, Néstor

- (2016). "The use of 'life-enabling' practices among waria. Vulnerability, subsistence and identity in contemporary Jogjakarta", en Susanne Hofmann y Adi Moreno (Eds.), Intimate Economies. Bodies, Emotions and Sexualities on the Global Market. Palgrave Mcmillan, 163-184.

- (2017). "Creo que nos están pidiendo morir... ellas quieren morir. Las dimensiones olvidadas de los programas de prevención y tratamiento de VIH llevados a cabo con las waria de Jogjakarta, Indonesia". Desacatos. Revista de Ciencias Sociales, 54.

Nyman, Mikaela (2007). Democratising Indonesia: The challenges of Civil Society in the Era of Reformasi. Copenhagen: NIAS Press.

Matthews, Kirstin R.W.; Ho, Vivian (2008).“The grand impact of Gates Foundation”. EMB Oreports, vol. 9, no. 5: 409-412.

Mcneil, Donald G. JR. (2008). “Gates Foundation's Influence Criticized”. Artículo publicado en The New York Times el 16 de Febrero de 2008. Último acceso, 23 de septiembre de 2015: http://www.nytimes.com/2008/02/16/science/16malaria.html?_r=0

Morin, Jack (2008). “'It's mutual attraction': Transvestites and risk of HIV Transmission in Urban Papua", en Leslie Butt y Richard Eves (Eds.), Making Sense Of AIDS: Culture, Sexuality, and Power in Melanesia. University of Hawai'i press, 41-59.

Oetomo, Dédé

- 2000."Masculinity in Indonesia: Genders, Sexualities and Identities in a Changing Society", en Richard Parker, et al. (Eds.), Framing the Sexual Subject: The Politics of Gender, Sexuality, and Power. Londres: University of California Press, 46-59.

— 2002. "Now you see it, now you don't: Homosexual Culture in Indonesia", International Institute for Asian Studies Newsletter, no. 29: 9.

Ong, Aihwa; Collier, Stephen J. (2008).Global Assemblages: Technology, Politics, and Ethics as Anthropological Problems. Wiley-Blackwell.

Petryna, Adriana

- 2002. Life Exposed. Biological Citizens after Chernobyl. Princeton: Princeton University Press.

- 2009. When Experiments Travel: Clinical Trials and the Global Search for Human Subjects. Princeton: Princeton University Press.

Pfeiffer, James; Nichter, Mark (2008). "What Can Critical Medical Anthropology Contribute to Global Health? A Health Systems Perspective". Medical Anthropology Quarterly, vol. 22, issue 4: 410-415.

Peletz, Michael P.

- 2006 "Transgenderism and Gender Pluralism in Southeast Asia Since Early Modern Times". Current Anthropology, 47(2): 309-340.

- 2011 "Gender Pluralism: Muslim Southeast Asia Since Early Modern Times". Social Research: An International Quarterly, 78(2): 659-686.

Pérez, Beatriz G. (ed.) (2012). Antropología y desarrollo. Discurso. Prácticas y actores. Madrid: La Catarata.

Pisani, E.; Girault, P.; Gultom, M.; et al. (2004). "HIV, syphilis infection, and sexual practices among transgenders, male sex workers, and other men who have sex with men in Jakarta, Indonesia”. Sexually Transmitted Infections, vol. 80(6): 536-540.

Prabawanti, C.; Bollen, L.; Palupy, R.; et al. (2011)."HIV, Sexually Transmitted Infections and Sexual Risk behaviour Among Transgenders in Indonesia”. AIDS and behavior, vol. 15(3): 663-673. 
Prats, Oriol J. (2006) “Teoría y práctica del desarrollo. Cambios en las variables de la 'ecuación del desarrollo’ en los últimos 50 años”. Revista del CLAD Reforma y Democracia, no. 36.

Rabeharisoa, Vololona (2006). "From representation to mediation: the shaping of collective mobilization on muscular dystrophy in France". Social Science \& Medicine, 62: 564-576

Reynolds, Susan W.; Van der Geest, Sjaak y Hardon, Anita (2003). Social lives of Medicines. Cambridge: Cambridge University Press.

Rose, Nikolas (1999). Powers of Freedom: Reframing Political Thought. Cambridge: Cambridge University Press.

Safitri, Dian M. (2013). "The politics of piety in the Pondok Pesantren Khusus Waria AlFattah Senin-Kamis Yogyakarta. Negotiating the Islamic religious embodiment", en Burhanudin, Jajat and Kees van Dijk (Eds.), Islam in Indonesia: Contrasting Images and Interpretations. Amsterdam: Amsterdam University Press, 91-108.

Sanahuja, José Antonio (2007). “Más y mejor ayuda? La Declaración de París y las tendencias en la cooperación al desarrollo", en Manuela Mesa (Coord.), Guerra y conflictos en el Siglo XXI: Tendencias globales. Anuario 2007-2008 del Centro de Educación e Investigación para la Paz (CEIPAZ). Madrid: CEIPAZ, 71-101.

Sen, Amartya (1989). "Development as capability expansion". Journal of Development Planning, 19(1): 41-58.

Singer, M.; Erickson, P.; Badiane, L.; et al. (2006). "Syndemics, sex and the city: Understanding sexually transmitted diseases in social and cultural context". Social Science \& Medicine, vol. 63: 2010-2021.

Sumner, Andy; Lawo, Thomas (2013). The post-2015 development agenda: A review of the debates and potential elements of a joint EU strategy. EADI Policy Paper Series.

Taha, Nur'Ain (2012). Let me be a servant of God: A study of Pondok Pesantren Khusus waria Senin-Kamis in Yogyakarta. Tésis de máster no publicada. Departamento de estudios sudasiáticos. Universidad Nacional de Singapore.

Towghi, Fouzieyha (2013). "The Biopolitics of Reproductive Technologies beyond the Clinic: Localizing HPV Vaccines in India". Medical Anthropology: cross-cultural Studies inHealth and Illness, 32(4): 325-342.

UNAIDS (2012). Republic of Indonesia: Country report on the Follow up to the Declaration of Commitment On HIV/AIDS (UNGASS). Reporting period 2011-2012. Indonesian National AIDS commission. 